\title{
Fuzzy Logic for Walking Patterns Based on Surface Electromyography Signals with Different Membership Functions
}

\author{
Nurhazimah Nazmi ${ }^{1,2}$, Yamamoto Shin-ichiroh ${ }^{2}$, Mohd Azizi Abdul Rahman ${ }^{1+}$, Siti Anom \\ Ahmad $^{3}$, Dimas Adiputra ${ }^{1}$, Hairi Zamzuri ${ }^{1}$ and Saiful Amri Mazlan ${ }^{1}$ \\ ${ }^{1}$ Vehicle System Engineering, Malaysia Japan International Institute of Technology, Universiti Teknologi \\ Malaysia, Jalan Sultan Yahya Petra, 54100 Kuala Lumpur, Malaysia \\ ${ }^{2}$ Department of Bio-science and Engineering, College of Systems Engineering and Science, Shibaura \\ Institute of Technology, Omiya Campus, Fukasaku 307, Saitama-city, Saitama-pref., 337-8570 Japan \\ ${ }^{3}$ Department of Electrical and Electronic Engineering, Faculty of Engineering, Universiti Putra Malaysia, \\ 43400 Serdang, Selangor, Malaysia
}

\begin{abstract}
Classifying walking patterns is important in developing assistive robotic devices, especially for lower limb rehabilitation. Recently, Fuzzy Logic (FL) controllers have successfully been applied in grasping and control system for upper limb based on surface Electromyography (EMG) signals. Therefore, this paper evaluates the performance of FL with different membership functions in discriminating walking phases (e.g, stance and swing phases). The accuracy of two widely used membership functions (MF) like triangular and Gaussian is compared to identify their behavior for detecting the phases of walking. In this study, the MATLAB and Simulink toolboxes are used to examine the performance of each MF. Our findings show Gaussian MF gained better performance than the triangular MF with $90 \%$ of classification accuracy. Therefore, the Gaussian MF could be the best solution to classify the walking phases in this work.
\end{abstract}

Keywords: walking phases, fuzzy logic, pattern recognition, classification.

\section{Introduction}

Walking pattern or also known as gait is one of the most common forms of human movement which can be considered as complex and individualistic. By delineating the walking phases would improve the functionality of robotic devices during rehabilitation. In 2012, the control algorithms for robotic systems in orthoses, prostheses, and exoskeletons especially adaptation to different walking conditions and gait phase identification has been extensively discussed in [1]. Walking patterns involving two main phases namely stance and swing. The stance phase (SP) starts when the heel strike passes through the midstance and ends by taking the toe off the ground to start the swing phase (SW). EMG is known as an electrical manifestation of neuromuscular activation that emanates from body muscles during contraction and/or relaxation. According to Rechy-Ramirezn and Hu [2], FL classifier had many advantages in the classification of EMG signals as this approach exploits tolerance of uncertainty, imprecision and partial truth, to achieve tractable, robust, and low-cost solutions. In robot grasping system, an FL classifier guarantees better results as they mimic the human behavior very well during the operation [3]. In addition, the MF is to be one of the factors that leads the decision making. Zaki et al. [4] used triangular and trapezoid MFs of FL to investigate the behavior of the gripper for robot grasping system. As a result, the triangular MF is easier and has faster computation time than trapezoid MF. In contrast, Ahmad et al. [5] identified that the computation time also can be faster for Gaussian MF in the sense of decision making. On the other hand, Vincent et al. claimed that the triangular

\footnotetext{
+ Corresponding author. Tel.: +60-322031463; fax: +603-22031266.

E-mail address: azizi.kl@utm.my.
} 
MF lacks of linguistic interpretation in comparison to the Gaussian approach [6]. For biosignal processing, trapezoid and triangular MFs of FL had been applied to detect the epileptiform activity in the electroencephalogram signals [7] and discriminate the stages of contractions of wrist muscles [8]. To our best knowledge, the performance of Gaussian MF of FL in biosignal analysis had not yet evaluated. Therefore, this paper attempts to examine the performance of Gaussian and triangular MFs in FL in detecting walking phases based on surface EMG signals. Then, we further explore the appropriateness of MF that could lead to a better decision making.

\section{Methodology}

\subsection{Data Acquisition}

To analyse the EMG signals, 9 healthy subjects with the mean age 25.8 years were recruited to undergo the experiment. The exclusion criteria for participants for this study are such as no history of lower extremity infirmity, or suffering from musculoskeletal disease/ nerve injury. All the EMG recordings were carried out by using EMG measurement device for example a two-channelled wireless EMG amplifier. The surface EMG signals were detected by placing the $\mathrm{Ag} / \mathrm{AgCl}$ bipolar electrodes and deployed with $2 \mathrm{~cm}$ interelectrode distance on gastrocnemius lateralis (GL). The procedures to place the electrode were referred to the Surface Electromyography for the Non-Invasive Assessment (SENIAM) [9]. In the experiment, each of the subjects walked bare-footed on treadmills within 60 seconds at a constant speed (level 2). The sampling frequency of surface EMG signals was $1250 \mathrm{~Hz}$ and sampled by using the AcqKnowledge software. The signals were segmented approximately $313 \mathrm{~ms}$ adjacent window for each SP and SW. Then, the signals were filtered using a low-pass Butterworth filter with a cut-off frequency of $6 \mathrm{~Hz}$ and full-wave rectified.

\subsection{Feature Extraction}

An investigation concerning to the suitability of PDF to represent the distribution of the sampled surface EMG signals becomes relevant since it can help to improve algorithms on onset detection which applied in biofeedback [10]. In this study, the features of surface EMG signals are extracted using probability distribution, more specifically a Cauchy distribution as presented in [11]. Given the sampled data of surface EMG signals, y the Cauchy distribution has the probability density function as of the following:

$$
f(y)=1 / \pi \alpha(1+(y-\alpha / \varepsilon) 2)
$$

where $\varepsilon$ is a location parameter and $\alpha$ is the scale parameter. The value of Cauchy's parameter is estimated using the Maximum Likelihood technique. This method selects the set values of surface EMG signals, $\mathrm{y}_{1, \ldots . ., \mathrm{y}_{\mathrm{n}}}$ of the model parameters and the log-likelihood is given by:

$$
l\left(\varepsilon, \alpha \mid \mathrm{y}_{1}, \mathrm{y}_{2} \ldots, \mathrm{y}_{\mathrm{n}}\right)=-\mathrm{n} \log (\alpha \Pi)-\sum_{\mathrm{i}=1}^{\mathrm{n}} \log \left(1+\left(\mathrm{y}_{\mathrm{i}}-\varepsilon / \alpha\right)^{2}\right)
$$

The estimated parameters will be obtained by differentiating eq. (2) and maximizes the likelihood function with respect to $\varepsilon$ and $\alpha$. In this work, parameters value were calculated using EasyFit software. As a result, the average values of $\varepsilon$ and $\alpha$ of 30 segmented of SP and SW for each subject were identified as shown in Fig. 1. From the figure, the value range of $\varepsilon$ during the SP lies between 0.0107 and 0.0174 . Meanwhile, for SW the range is from 0.0026 to 0.0033 . The minimum values of $\alpha$ during the SP and SW are 0.0063 and 0.0010 respectively. For maximum value of $\alpha$ during the SP and SW are 0.0141 and 0.0018 respectively.

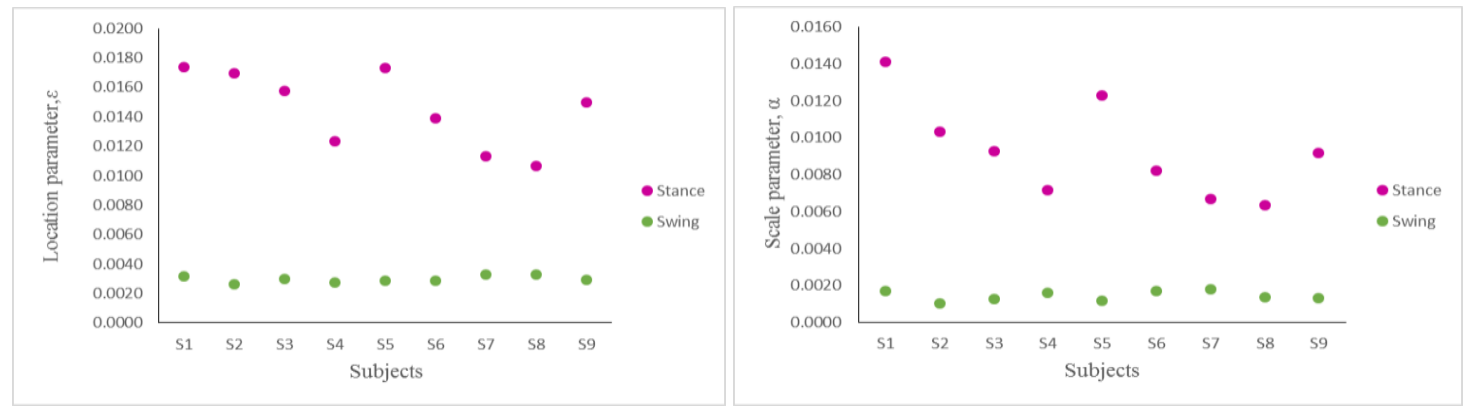

Fig. 1: Average parameters value of the Cauchy distribution. 


\subsection{Classification}

A Fuzzy Logic (FL) classifier will be used as a function to map the pattern and match appropriately the extracted features in determining the final output. These output states represent the SP and SW. The use of FL classifier is well known in providing a simple way and has less overshoot to arrive at a definite conclusion [12]. In this study, the value of $\varepsilon$ and $\alpha$ will be the inputs while the output is the walking phases. Basically, the FL classifier consists of MFs and the fuzzy inference system. For fuzzification, two fuzzy set boundaries are designed for $\varepsilon$ and $\alpha$ in which the range between 0.0026 and 0.0174 for SP and 0.0010 to 0.0141 for SW. For the output, the walking phases have also two fuzzy sets; SP and SW which ranges between 0 to 0.2 for triangular and Gaussian MFs. By using the Mamdani technique in FL toolbox and Simulink, the rules are evaluated to allocate the walking phases. Notice that the rules are constructed to discriminate the SP and SW in the specified value. The values of $\varepsilon$ during the SP are in the range from 0.0107 to 0.0174 and during the SW are between 0.0026 and 0.0033 . Besides, the values of $\alpha$ for the SP lies between 0.0063 to 0.0141 and 0.0010 to 0.0018 for the SW. The rules of FL are; 1) IF $\varepsilon$ is high AND $\alpha$ is high THEN SP, 2) IF $\varepsilon$ is low AND $\alpha$ is low THEN SW. Lastly, defuzzification process determines the output of the system. The FL classification is developed using MATLAB's FL toolbox and tested using Simulink to simulate the performance of the designed classification. Figs. 2 and 3 show the input and output of triangular and Gaussian MFs respectively.
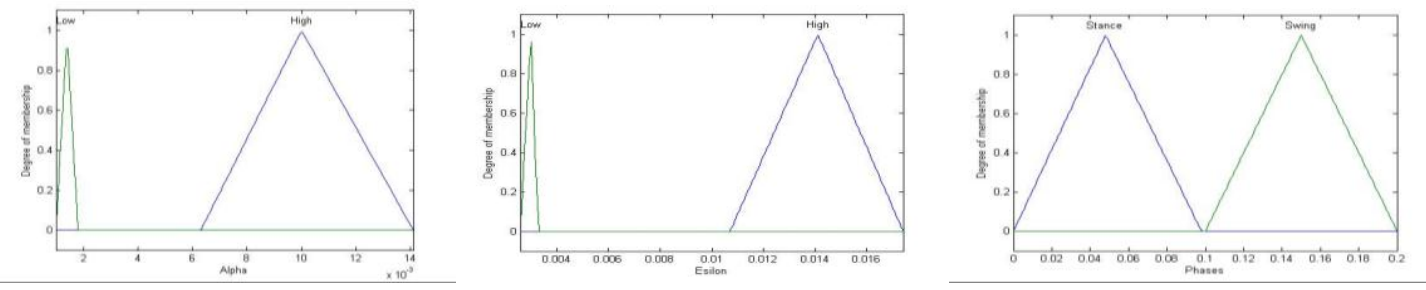

Fig. 2: Inputs and output of triangular-type membership functions.
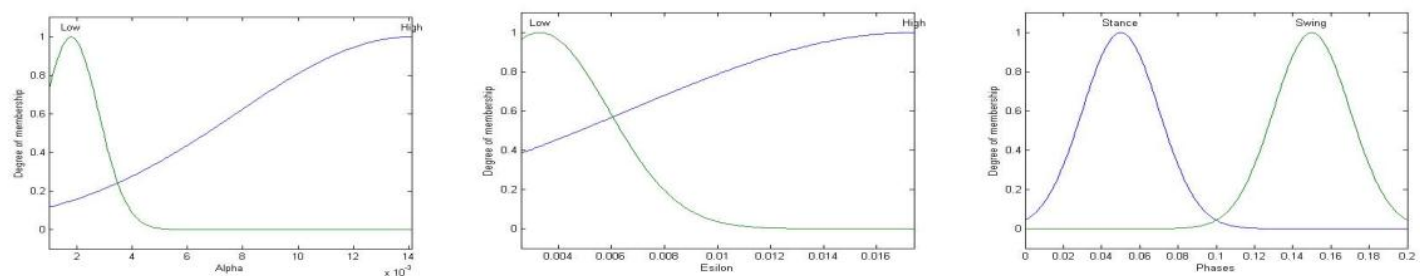

Fig. 3: Inputs and output of Gaussian-type membership functions.

\subsection{Accuracy}

The work presented in this paper only focuses on two phases of walking gaits. Although the fuzzy rules seem very simple but it provides a better performance by choosing appropriate MFs as demonstrated in [5]. Then, this work examines the accuracy of both systems by the following equation:

$$
\text { Accuracy }=(\text { number of correct classification /number of total classifications }) \times 100 \%
$$

\section{Results and Discussion}

The performance of the proposed system using triangular and Gaussian MF was carried out by using 10 segmented surface EMG signals of another 8 subjects. The estimated value of $\varepsilon$ and $\alpha$ were implemented in the designated Simulink blocks and the output of the FL with the triangular and Gaussian MFs were observed. All results are shown in Table 1 which S1 (Subject 1), S2 (Subject 2), S3 (Subject 3), S4 (Subject 4), S5 (Subject 5), S6 (Subject 6), S7 (Subject 7), S8 (Subject 8), S9 (Subject 1) and S10 (Subject 8). Referring to Table 1, the output values from the Gaussian and triangular MFs for S1 and S2 were similar which is 0.05 and 0.049 respectively, this is the SP. A similar result for S8, the MF describes the walking phases as the stance. As we can see, both MFs locate S5, S6, S7 and S10 in the SP area. Despite that, the triangular MF obtained an output value that categorized the signals as SW for S3 and S9. Contrary with the Gaussian MF, it categorized both signals as stance phase. In other words, Gaussian MF accurately classifies the phases of walking. Nonetheless, the output value for S4 shows the triangular MF provide a correct walking phase instead of Gaussian MF. The main reason to this results due to the interpretation of linguistic variable as the triangular MF cannot appropriately simulate the variable [5] which may have adverse effects 
on operational and control decisions [6]. In particular, surface EMG signals were correctly classified with 9 and 8 out of 10 signals for Gaussian and triangular MFs respectively. In other words, the triangular MF gained $80 \%$ of classification accuracy while the Gaussian MF achieved $90 \%$ of classification accuracy.

Table 1. Classification of EMG Signals

\begin{tabular}{cccccc}
\hline EMG Signals & $\varepsilon$ & $\alpha$ & Phases & Gaussian & Triangular \\
\hline S1 & 0.01199 & 0.00681 & Stance & 0.05 & 0.049 \\
S2 & 0.01358 & 0.00811 & Stance & 0.05 & 0.049 \\
S3 & 0.00971 & 0.00537 & Stance & 0.051 & 0.1 \\
S4 & 0.01387 & 0.00593 & Swing & 0.05 & 0.1 \\
S5 & 0.00355 & 0.00164 & Swing & 0.09 & 0.1 \\
S6 & 0.00307 & 0.00164 & Swing & 0.09 & 0.15 \\
S7 & 0.00414 & 0.00154 & Swing & 0.09 & 0.1 \\
S8 & 0.0166 & 0.01032 & Stance & 0.05 & 0.05 \\
S9 & 0.02278 & 0.01172 & Stance & 0.05 & 0.1 \\
S10 & 0.00234 & 0.000787 & Swing & 0.09 & 0.1 \\
\hline
\end{tabular}

\section{Conclusion and Future Works}

This paper designed FL classifier with triangular and Gaussian MFs based on estimated parameter values of Cauchy distribution from 9 healthy subjects. The accuracy of both MFs were examined by using another 10 segmented signals of 8 subjects. As a result, detecting walking phases using FL with Gaussian MF performed better than triangular MF in this approach. Even though previous researchers has used the triangular MF for their system, the Gaussian MF can be considered in pursuing their expected outcomes.

\section{Acknowledgements}

The work presented in this study is funded by Ministry of Education Malaysia and Universiti Teknologi Malaysia under research university grant. VOTE NO: 09H90.

\section{References}

[1] R. Jiménez-Fabián and O. Verlinden. Review of control algorithms for robotic ankle systems in lower-limb orthoses, prostheses, and exoskeletons. Medical Engineering and Physics 2012, 37, 505-511.

[2] E. J. Rechy-Ramirezn and H. Hu. Bio-signal based control in assistive robots: a survey. Digital Communications and Networks 2015, pp. 85-101.

[3] W. Xia. R. Alberto. Fuzzy Logic In Robot Grasping Control. IEEE/RSJ International Workshop on Intelligent Robots and Systems IROS 1991, pp. 1632 - 1637.

[4] A .M. Zaki, O .A . Mahgoub, A .M. El-Shafei and A .M. Soliman. Control of Efficient Intelligent Robotic Gripper Using Fuzzy Inference System. Fuzzy Inference System - Theory and Applications, Intech Open, 2012, 85-112.

[5] H. Ahmad, S. Razali, and M. R. Mohamed Fuzzy Logic Controller Design for A Robot Grasping System with Different Membership Functions. IOP Conf. Series: Materials Science and Engineering, 2013, 53: 1-8.

[6] V. O. S. Olunloyo, A. M. Ajofoyinbo and O. Ibidapo- Obe. On Development of Fuzzy Controller: The Case of Gaussian and Triangular Membership Functions. Journal of Signal an d Information Processing, 2011, 2:257-265.

[7] C. J. James, R.D.Jones,P.J. Bones,G.J.Carroll. Detection of epileptiform discharges in the EEG by a hybrid system comprising mimetic,self-organized artificial neural network and fuzzy logic stages. Clin. Neurophysiol,1999.

[8] S. A. Ahmad, A. J. Ishak and S. Ali. Classification of Surface Electromyographic Signal Using Fuzzy Logic for Prosthesis Control Application. EMBS Conference on Biomedical Engineering \& Sciences, 2010, 471-474.

[9] D. F. Stegeman , H. J. Hermens. European Recommendations for Surface ElectroMyoGraphy: Results of the SENIAM Project (CD-rom)," The Netherlands: Roessingh Research and Development, 1999.

[10] S. Thongpanja, A. Phinyomark and C. Limsakul. and Phukpattaranont, P. Probability Density of Electromyography Signal for Different Levels of Contraction of Biceps Brachii. 2013.

[11] I. D. G. Rosa, M. A. C. Garcia and M. N. D, Souza. Investigation of probability density functions in modeling sample distribution of surface electromyographic (sEMG) signals. Archives of Control Sciences, 2013,23:381-393.

[12] I. H. Altas, and J. Neyens. A Fuzzy Logic Decision Maker and Controller for Reducing Load Frequency Oscillations in Multi-Area Power Systems. IEEE Power Engineering Society General Meeting, pp. 1-9, 2006. 